\title{
Will an increased supply of undertrained, under-resourced surgeons solve our wait-list problems?
}

\author{
Edward W Buchel MD, Editor-in-Chief
}

$\mathrm{T}$ he balancing act between supply and demand requires continuous effort, regardless of the profession that one examines. Predicting workforce requirements is becoming increasingly critical as regions struggle with the costs of training and subsequently employing surgeons. Currently, a discrepancy exists between the number of trained surgical specialties graduates and the number possessing adequate employment within their specialty. While not as publicized as orthopedics, plastic surgery will also need to look carefully at the number of residency spots that the country has as many of our graduates struggle to find suitable employment.

Plastic surgery continues to be a highly skilled, technical surgical subspecialty requiring years of practice and ongoing skill maintenance. While a generalization, the idea that 'the more you do, the better you get at it' still rings true for much of what we do. With this in mind, I continue to be dismayed by the limited access to operating room time that awaits most of our graduating residents and fellows on entering the surgical workforce. Residency programs continue to limit resident duty hours, condensing the time for developing surgical skills while in training programs. Subsequently, they enter a system in which regional cost containment is achieved by limiting access to operative resources needed to care for surgical patients. Surgical skills continue to languish because case volumes are limited. The resulting effect on wait lists and patient care is negative. Plastic surgery leaders must actively advocate for appropriate operative resources for each graduating surgeon BEFORE increasing training spots, which may further dilute these limited resources. The more we restrict graduates' access to operating rooms, the more we delay optimization of their surgical skills, decreasing their efficiency, increasing surgical complications and, in all probability, decreasing their desire to deliver complex surgical care.

Currently, in many areas of the country, operating room access has been limited to the point that surgeons are unable to generate income billing fees for service, while patients on wait lists go untreated. Graduating plastic surgeons are now being recruited to hospitals and regions with guaranteed incomes BUT limited operative resources. Each region can then claim they have specialty surgeons available, but limited care is delivered. This makes little sense. Instead of providing adequate resources to each surgeon so they can deliver care, develop their skills quickly and generate income based on care delivered, they are essentially being paid to wait. This is very concerning for our specialty. If we continue to graduate surgeons who will take increasingly longer to develop their skills, costs per case for these surgeons will continue to be higher for longer periods of time and the pressure to limit their access to resources will increase. Regional cost containment is an over-riding theme in almost every aspect of medicine and expensive surgical specialties are frequently under scrutiny. While privately paying patients are more of an option for plastic surgeons than most other specialties, this should not be used as a safety net for these surgeons to generate incomes and develop their skills.

Predicting plastic surgery demand a decade from now will always be challenging. Striving to accurately supply our population with highly skilled surgeons is what our training programs and specialty aims for. While doing so, the argument against oversupplying new plastic surgeons and under-resourcing the ones we graduate needs to be kept in mind. 